\title{
Prospects for detecting stellar tidal disruptions with LOFAR
}

\section{Sjoert van Velzen*}

IMAPP, Radboud University Nijmegen, The Netherlands

E-mail: s.vanvelzen@astro.ru.nl

\section{Heino Falcke}

IMAPP, Radboud University Nijmegen, The Netherlands

ASTRON, Dwingeloo, The Netherlands

\section{Glennys R. Farrar}

Center for Cosmology and Particle Physics \& Department of Physics, New York University, USA

\begin{abstract}
A star that passes too close to a super-massive black hole is shredded and an electromagnetic flare is emitted as the bound fraction of the stellar debris falls back onto the black hole. Candidate examples of such accretion flares have been observed at optical to X-ray frequencies. If a jet is launched from the accretion disk, tidal flares might be detectable at radio frequencies too. Here we estimate the luminosity of this jet using the observed properties of a sample of (candidate) tidal flares that have been found in SDSS and GALEX data. The scaling between disk and jet luminosity as observed for radio-loud quasars is used to predict a jet flux of $\sim 0.1 \mathrm{mJy}$. This flux is well observable with LOFAR and other radio telescopes.
\end{abstract}

ISKAF2010 Science Meeting - ISKAF2010

June 10-14, 2010

Assen, the Netherlands

${ }^{*}$ Speaker. 


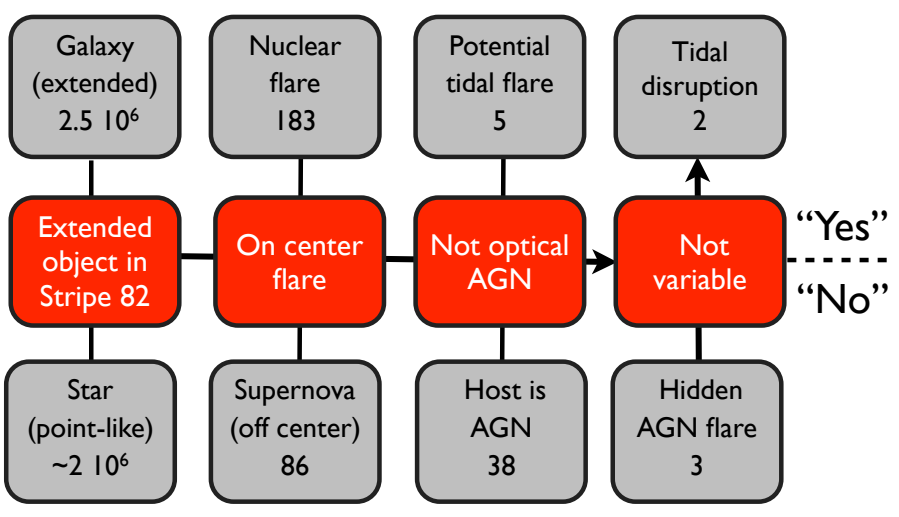

Figure 1: Flow chart summarizing the successful search for tidal disruption flares in SDSS multi-epoch imaging data (Stripe 82) [1].

\section{Introduction}

When a star passes too close to a super-massive black hole, the tidal pull exceeds the selfgravity, and the star is disrupted. For black holes with mass $\lesssim 10^{8} M_{\odot}$, the tidal disruption radius lies outside the Schwarzschild radius and an electromagnetic flare can be observed as the debris falls back onto the black hole [2,3]. These flares are an important probe to black holes in quiescent galaxies; a large sample will enable a census of black holes including otherwise undetectable mass ranges.

A number of (candidate) examples of tidal disruption events (TDE) have been identified in UV and X-ray surveys $[4,5,6,7,8]$. Recently, two TDEs have been identified using optical data [1]. In the following section we will briefly summarize this work. In section 3 we use the observed properties of these flares to estimate the luminosity of jets from tidal disruptions.

\section{Tidal disruption flares found in optical data}

Two TDE have been found in $\sim 300 \mathrm{deg}^{2}$ of multi-epoch imaging data of the Sloan Digital Sky Survey (SDSS), also known as "Stripe 82" [10, 11]. This dataset contains over 2 million galaxies, each observed about 70 times over a nine year baseline. This rather large sample of galaxies, with many having spectra, enables flares to be classified into well-defined categories. Supernovae are identified by being significantly off-center from their host. Flares from active galactic nuclei

\begin{tabular}{ccccc}
\hline \hline ID & $\begin{array}{c}T \\
\left(\times 10^{4} K\right)\end{array}$ & $\begin{array}{c}L_{g} \\
\left(\times 10^{43} \mathrm{erg}\right)\end{array}$ & $\begin{array}{c}L_{\mathrm{BB}} \\
\left(\times 10^{43} \mathrm{erg}\right)\end{array}$ & $z$ \\
\hline TDE1 & 2.4 & 0.5 & 3 & 0.138 \\
TDE2 & 1.8 & 4.1 & 10 & 0.252 \\
D1-9 & 5 & 0.2 & 14 & 0.326 \\
\hline \hline
\end{tabular}

Table 1: Observed properties of TDE1,2 identified in SDSS data [1]. We also show D1-9, a (candidate) TDE discovered in GALEX [9]. Columns list temperature, $g$-band luminosity, black body luminosity and redshift. 


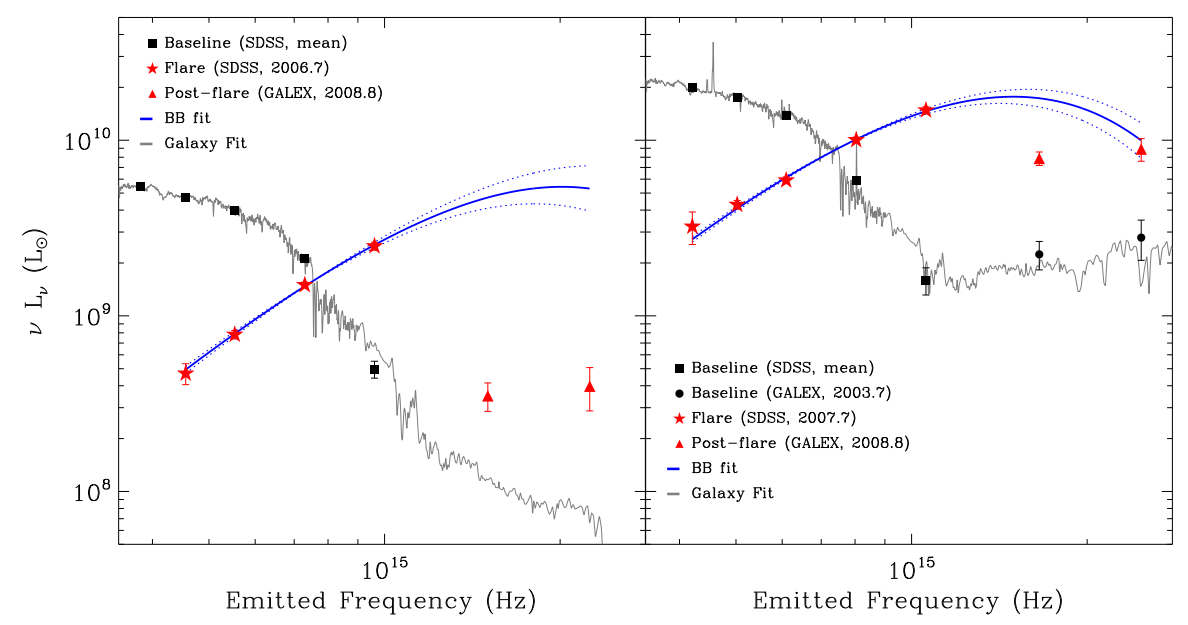

Figure 2: The SED of the two tidal flares discovered in SDSS data [1]. The optical baseline flux of the host is shown (black squares) with the best-fit combination of eigen spectra (grey line) [12]. The flux of the flare in the difference image (red stars) is well-fit by a black body (blue, with dotted lines indicating the 1- $\sigma$ uncertainly)

(AGN) can be identified by the additional variability they display in the seasons beyond the main flare. The search pipeline of [1] is summarized in Fig. 1. The spectral energy distributions (SED) of the two TDE (dubbed "TDE1" and "TDE2") are shown in Fig. 2. In Table 1 we summarize the observed properties of TDE1,2.

\section{Jet prediction}

As the debris of the disrupted star is accreted, a jet may be launched. To estimate the luminosity of this jet, we use the jet-disk relation that has been observed for radio-loud and radio-quiet quasars [13]. Here we take the expected radio flux of the radio cores of radio-loud quasar jets, which is applicable to at least $10 \%$ of the black holes. The assumed radio core spectrum we take is flat and steady and we note that individual flares might be more luminous and show peaked radio spectra that travel through frequency. We conservatively obtain the disk luminosity from the black body (BB) luminosity of the three TDE presented in Table 1. We find that for these three flares, the predicted jet flux is about $0.4 \mathrm{mJy}$. For comparison we also consider a "maximal" scenario for the

\begin{tabular}{lcc}
\hline \hline & based on observed BB luminosity & maximal \\
\hline$F_{\text {jet }}$ & $0.4 \mathrm{mJy}$ & $1-7 \mathrm{mJy}$ \\
$t_{\mathrm{obs}}(\tau=1)$ & $0.5-1 \mathrm{yr}$ & $2 \mathrm{yr}$ \\
$t_{\text {int }}(3 \sigma)$ & $3 \mathrm{~h}$ & $1-20 \mathrm{~min}$ \\
\hline \hline
\end{tabular}

Table 2: Predicted jet properties of the three TDE considered (Table 1) for two different scenarios (see section 3). These results were calculated using an angle between the jet and observer of $30 \mathrm{deg}$ and a jet Lorentz factor of 5. The first row lists the observed flux of the jet, the second row lists the time the jet becomes optically thick at $120 \mathrm{MHz}$, and the last row lists the integration time for a 3- $\sigma$ detection with LOFAR. 
disk luminosity. We assume that $0.5 M_{\odot}$, the bound material from the disruption, is accreted in one year and the disk radiates at $1 \%$ efficiency. In this case, the jet luminosity equals $3 \times 10^{30} \mathrm{erg} \mathrm{s}^{-1}$ for any TDE and the observed flux depends on the distance only. In Table 2 these findings are summarized.

\section{Discussion}

We have presented an estimate of the jet flux from known tidal flares of $\sim 0.1 \mathrm{mJy}$. This flux is easily accessible for most radio observatories; follow-up observations of TDE at radio frequencies will allow us to test the hypothesis that jets are launched during the accretion of the disrupted star. The potential for detecting TDE in a multi-epoch radio survey without higher frequencies servering as a trigger will be discussed elsewhere.

\section{References}

[1] van Velzen, S. et al. Optical discovery of stellar tidal disruption flares (2010). Submitted.

[2] Hills, J. G. Possible power source of Seyfert galaxies and QSOs. Nature 254, 295-298 (1975).

[3] Rees, M. J. Tidal disruption of stars by black holes of 10 to the 6th-10 to the 8th solar masses in nearby galaxies. Nature 333, 523-528 (1988).

[4] Bade, N., Komossa, S. \& Dahlem, M. Detection of an extremely soft X-ray outburst in the HII-like nucleus of NGC 5905. Astron. \& Astrophys. 309, L35-L38 (1996).

[5] Donley, J. L., Brandt, W. N., Eracleous, M. \& Boller, T. Large-Amplitude X-Ray Outbursts from Galactic Nuclei: A Systematic Survey using ROSAT Archival Data. Astron. J. 124, 1308-1321 (2002).

[6] Esquej, P. et al. Evolution of tidal disruption candidates discovered by XMM-Newton. Astron. \& Astrophys. 489, 543-554 (2008).

[7] Cappelluti, N. et al. A candidate tidal disruption event in the Galaxy cluster Abell 3571. Astron. \& Astrophys. 495, L9-L12 (2009).

[8] Gezari, S. et al. Luminous Thermal Flares from Quiescent Supermassive Black Holes. Astrophys. J. 698, 1367-1379 (2009).

[9] Gezari, S. et al. UV/Optical Detections of Candidate Tidal Disruption Events by GALEX and CFHTLS. Astrophys. J. 676, 944-969 (2008).

[10] Frieman, J. A. et al. The Sloan Digital Sky Survey-II Supernova Survey: Technical Summary. Astron. J. 135, 338-347 (2008).

[11] Abazajian, K. N. et al. The Seventh Data Release of the Sloan Digital Sky Survey. Astrophys. J. Suppl. 182, 543-558 (2009).

[12] Blanton, M. R. \& Roweis, S. K-Corrections and Filter Transformations in the Ultraviolet, Optical, and Near-Infrared. Astron. J. 133, 734-754 (2007).

[13] Falcke, H., Malkan, M. A. \& Biermann, P. L. The jet-disk symbiosis. II. Interpreting the radio/UV correlations in quasars. Astron. \& Astrophys. 298, 375-394 (1995). 